\title{
Gigabit Laser Ethernet Transceiver for Free-Space Optical Communication Systems
}

\author{
Julio Ramírez, Amita Shrestha, Swaminathan Parthasarathy, Dirk Giggenbach \\ German Aerospace Center (DLR),Muenchner Str. 20, 82234 Wessling, Germany \\ Corresponding author e-mail address: Julio.Ramirez@dlr.de
}

\begin{abstract}
This paper presents a novel FPGA-based Gigabit Laser Ethernet Transceiver featuring a custom designed transmission protocol capable of overcoming the inherent impairments of FSO communications. The prototype is characterized and validated with a ground to ground test. OCIS codes: (060.2605) Free-space optical communications; (060.4259) Networks, packet-switched.
\end{abstract}

\section{Introduction and motivation}

Free Space Optical (FSO) communication is an emerging technology combining several advantageous characteristics, like: potential of highest data rates (several gigabits per second), small antenna (telescope) sizes combined with highest antenna gain, tap-proofness, working in the license-free near-infrared spectrum bands. FSO links have applications in various scenarios, from short-distance ground-ground links (in scenarios where cable links are financially or technically impractical) to mid-range and mobile scenarios (several kilometers) like aeronautic reconnaissance downlinks from observation platforms that are monitoring traffic, industrial/infrastructure systems, or natural disasters, to long-range links (several hundred kilometers) like between stratospheric platforms or satellite downlinks, up to telemetry downlinks from deep-space probes [1], [2], [3]. In this regard, the increasing need for data-throughput of current and near-future high resolution sensor systems have further enhanced the potential of such applications and have increased the demand for higher data rates, requiring a migration of the data link technology from conventional RF-links to FSO.

To date, data-rates higher than $1 \mathrm{Gbps}$ have been demonstrated by rather simple single-channel optical communication links, with the potential to be increased up to 10Gbps with state-of-the-art technology and beyond that by Wavelength-Division-Multiplexing (WDM) technology. While with short-range links the dominant atmospheric impact onto the link is basically the increased signal attenuation by fog, clouds, and rain events, at midrange and above the scintillation effects caused by the index-of-refraction-turbulence (IRT) of the atmosphere [4] become a significant source of signal fading. Additionally, in mobile applications imperfect pointing and tracking performance is a second cause for fading. This leads to special requirements for providing a stable and robust FSO data link in such scenarios. Standard high-speed wired transmission formats must therefore be replaced by fadingrobust formats combined with error correction schemes, to compensate losses during fades.

The Optical Communication (OCG) Groups within the Institute of Communications and Navigation of the German Aerospace Center (DLR) are extensively involved in theoretical analysis as well as in the development and pre-operational implementation of advanced technologies for high speed laser communication between satellites, aircrafts, high-altitude platforms and ground stations. One of the typical applications is data downlink from a research aircraft (a Do-228) to the different optical ground stations. During this process, DLR has developed its own flight terminals, and fixed (OGS-OP) and transportable (TOGS) optical ground stations [5].

In most network applications worldwide, data are transmitted using the Ethernet Standard. However, the Ethernet frame structure is not suitable for mobile FSO communication scenarios, where a high fraction of long burst errors lead to high error rates and signal outages [6]. In order to tackle these issues, DLR has developed a line of FPGA-based Laser Ethernet Transceivers (LET) capable of establishing optical communication links with datarates up to $1.25 \mathrm{Gbps}$ and in full compliance with the IEEE 802.3 Ethernet Standard. Moreover, the LET processes the Ethernet data stream and arranges it to a more robust frame format protocol, and vice versa. While first implementations used delayed frame-retransmission as a means to protect the link against fade outages, subsequent versions use a custom LET frame format and advanced error protection techniques. This paper firstly describes the FSO communication protocol implemented as part of the current LET prototype and presents the characterization results obtained during a recent validation campaign.

\section{FSO Communication Protocol}

The block diagram detailing how data is transmitted from one Ethernet partner to another using the LET is shown in Fig 1. 


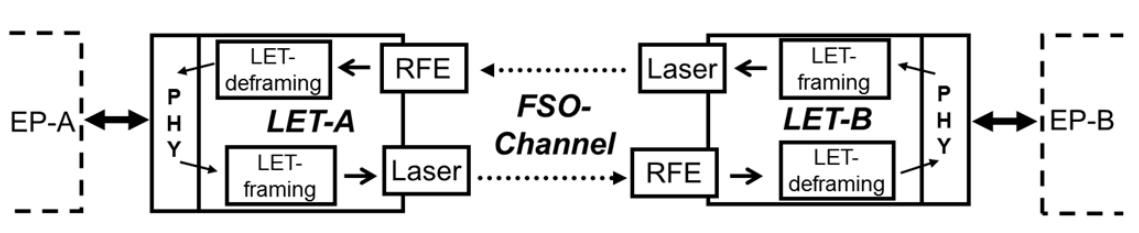

Fig 1. Transparent Optical Communication Link between two Ethernet Partners using LET

As shown on the diagram, the LET implements the Ethernet Physical Layer including a custom designed flow control to prevent data loss due to congestion or disruption of the optical link. As aforementioned, Ethernet frames are not suitable for FSO optical communications because long data frames are susceptible to bit-slips that cannot be corrected by simple error-correction techniques [3]. Therefore, as part of the implemented FSO protocol, Ethernet frames are arranged into special LET frames which are smaller and more robust (see Fig. 2).

\begin{tabular}{|l|l|l|l|l|}
\hline Frame Start & LF No. & Data size & Data & CRC \\
\hline
\end{tabular}

Fig. 2. Structure of the LET Frames

The LET frames contain information like start of the frame with synchronization bits to overcome the effect of scintillation thereby easing their re-synchronization at the receiver, an identifier for rearranging the Ethernet frames at the receiver (LF No.), size of payload data in the LET frame (Data size), data payload and CRC check bits. This robust LET frames are then converted from electrical to optical domain and then transmitted via laser to the partner terminal. At the receiver side, a receiver front-end (RFE) converts the received optical signal to electrical domain, then the LET prototype acquires and processes the LET frame, regenerates the Ethernet frames and finally forwards them to the data sink.. In addition, LET supports independent bidirectional link meaning uplink and downlink can work at different data rates.

\section{Characterization Setup and Results}

The experimental setup used to characterize the LET prototype is shown in Fig. 3. As seen, a FSO ground to ground link between an optical transmitter and the TOGS was established. The link distance was $4.1 \mathrm{~km}$. Two different Network Performance Analyzers were used, an Anritsu MD1230 at the transmitter side configured to transmit different Ethernet data streams with frame lengths from 64 to 1518 bytes, and an MP1590 at the receiver side configured to monitor the maximum throughput for different Ethernet frame lengths, thereby characterizing its performance and validating the LET's compliance with the IEEE 802.3 Ethernet Standard. Then, the MD1230 was configured to transmit Ethernet frames with randomly defined lengths between 64 and 1518 bytes, and accordingly the maximum throughput was measured at the receiver side. Fig. 4 summarizes the obtained results.

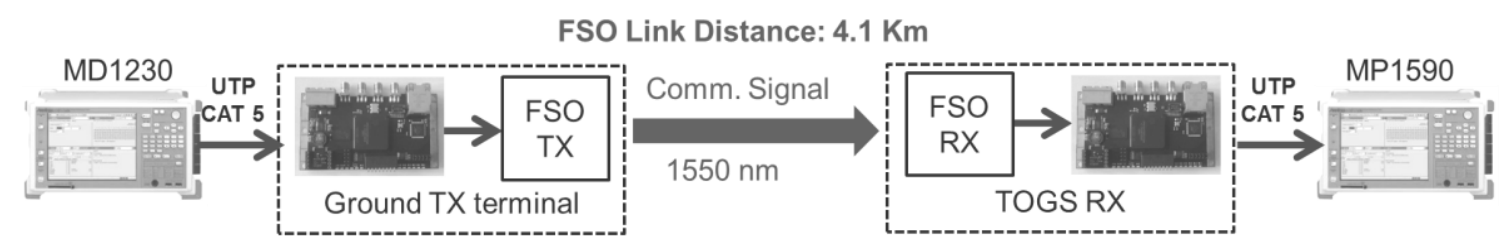

Fig. 3. Measurement Setup with custom LET-FPGA boards

As shown in Fig. 4, the LET prototype presents a penalty in terms of maximum achievable throughput when compared to the reference performance provided by the IEEE 802.3 Ethernet Standard. This condition can be easily quantified by comparing the maximum throughput achieved when transmitting Ethernet frames with fixed and randomly defined length. Specifically, the maximum throughput measured for a fixed frame length scenario is 873 Mbps, which corresponds to a penalty of circa $11.55 \%$, whereas for a randomly defined frame length the maximum throughput is $844.7 \mathrm{Mbps}$ for a penalty of circa $13.35 \%$. The throughput gap between the LET prototype and the IEEE Standard reference is due to the overhead introduced by the FSO communication protocol. Moreover, it should 
be noticed that this penalty is larger for shorter Ethernet frames and therefore the larger throughput difference for the randomly defined frame length scenario.

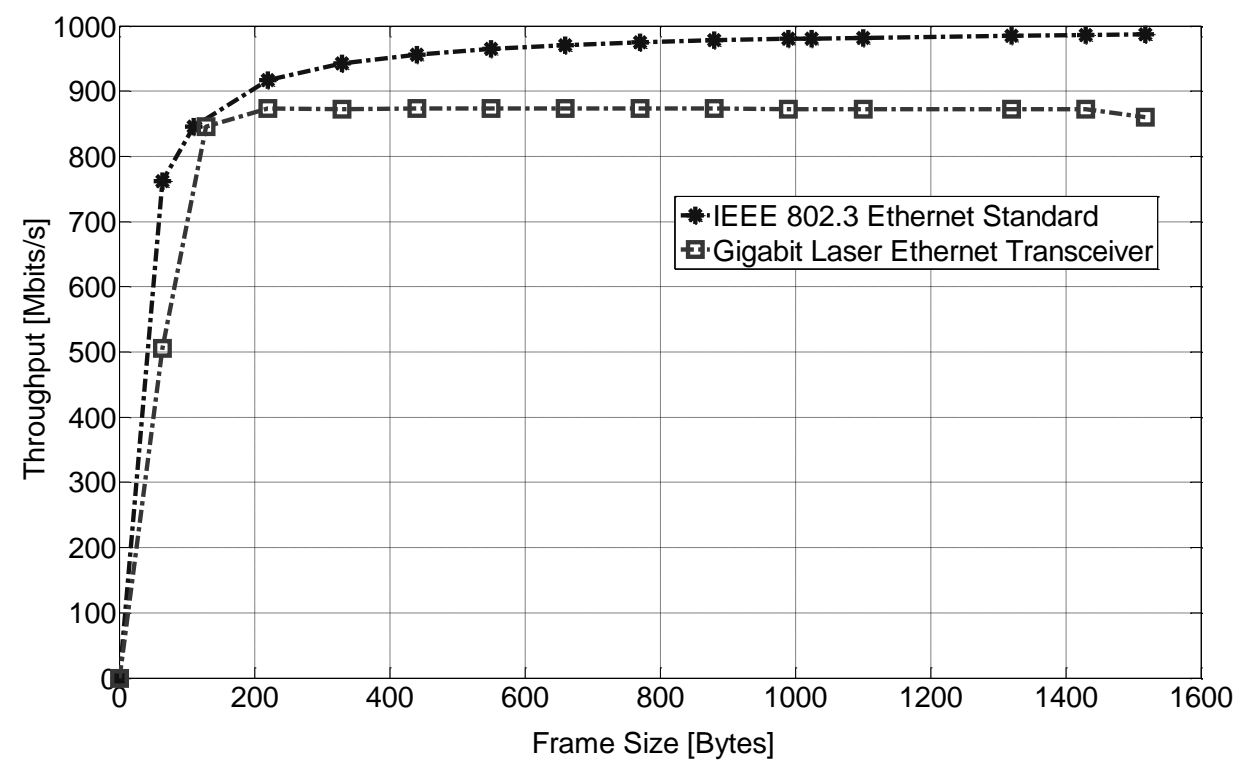

Fig. 4. Throughput measurement results

\section{Conclusion and Outlook}

A Gigabit LET for FSO communication systems, compliant with the IEEE 802.3 Ethernet Standard, has been demonstrated. In particular, the performance of its proprietary FSO communication protocol has been successfully validated with a ground to ground FSO link, featuring maximum throughput with penalties between $11 \%$ and $14 \%$ when compared with the reference throughput values.

The mean power received in mobile link scenarios varies strongly due to range-variations and varying atmospheric attenuation. In such cases, it is beneficial to lower down the data-rate instead of breaking down the complete communication link. Therefore, LET is foreseen to work at variable data-rates [7].

\section{Acknowledgements}

The authors would like to thank OCG group members of DLR. We also appreciate the support and assistance of the Institute for Telecommunication Research of University of South Australia during the development of the project.

\section{References}

[1] J. Horwath and C. Fuchs, Aircraft to Ground unidirectional laser-Comm. Terminal for High Resolution Sensors, in Free-Space Laser Communication Technologies XXI, ol 7199 (2009).

[2] F. David, D. Giggenbach, H. Henniger, J. Horwath, R. Landrock, and N. Perlot, Design Considerations for Optical Inter - HAP Links, in Proceedings ICSSC 2004 (2004).

[3] D. Giggenbach and H. Henniger, Fading-loss assessment in atmospheric free-space optical communication links with on-off keying, Optical Engineering (2008).

[4] L. C. Andrews and R. L. Philips, Laser Beam Propagation through Random Media (SPIE- The International society for Optical Engineering, 2005).

[5] A. Shrestha and M. Brechtelsbauer, Transportable optical ground station for high-speed free-space laser communication, in SPIE. Laser Communication and Propagation through the Atmospher and Oceans (2012).

[6] J. Perez, Z. Ghassemlooy, S. Rajbhandari, M. Ijaz, and H. Minh, Ethernet FSO Communications Link Performance Study Under a Controlled Fog Environment, Communications Letters, IEEE 16, 408-410 (2012).

[7] N. Perlot and T. D. Cola, Throughput Maximization of Optical LEO-Ground Links, in SPIE, Free-Space Laser Communication Technologies XXIV (2012). 